(2) Open Access Full Text Article

CASE REPORT

\title{
A spectrum of cutaneous toxicities from erlotinib may be a robust clinical marker for non-small-cell lung therapy: a case report and literature review
}

This article was published in the following Dove Press journal:

OncoTargets and Therapy

23 April 2015

Number of times this article has been viewed

Feng Jin'

Hui Zhu²

Li Kong ${ }^{2}$

Jinming $\mathrm{Yu}^{2}$

'Department of Radiation Oncology, Shandong Cancer Hospital and Institute, Shandong Academy of Medical Sciences, University of Jinan, Jinan, People's Republic of China; ${ }^{2}$ Department of Radiation Oncology, Shandong Cancer Hospital and Institute, Jinan, People's Republic of China
Abstract: Some literature suggests that an EGFR inhibition-induced rash can be used as a clinical marker, but few studies report the correlation between a spectrum of cutaneous toxicities from EGFR inhibition and drug efficacy. We report about a woman with a stage IV lung adenocarcinoma using erlotinib monotherapy, who experienced a spectrum of cutaneous toxicities, including papulopustular rash, mucositis, pruritus, xerosis, paronychia, and facial hirsutism. With treatment, her metastatic lesions shrunk remarkably. This report suggests that some non-small-cell lung cancer patients experiencing a spectrum of cutaneous toxicities might have a good tumor response using erlotinib monotherapy. Our findings may provide a method for clinicians to predict erlotinib efficacy in non-small-cell lung cancer therapy without knowledge of the EGFR mutation status.

Keywords: cutaneous toxicity, epidermal growth factor receptor inhibition, erlotinib, clinical marker, non-small-cell lung cancer

\section{Introduction}

In recent years, EGFR inhibition has been widely used for the treatment of patients with various solid tumors, including non-small-cell lung cancer (NSCLC). It has resulted in valuable clinical responses, but has also resulted in varying adverse effects. Cutaneous events represent the more relevant toxicities in terms of frequency and patient discomfort. ${ }^{1}$ Commonly experienced cutaneous adverse effects include papulopustular rash, perifollicular xanthoma, xerosis cutis/pruritus, eczema craquele, fissures/rhagades, paronychia, hypertrichosis and hair follicle abnormalities. ${ }^{2}$ Incidences of these adverse effects are frequent, and approximately $36 \%$ of patients show mucositis and $80 \%$ show papulopustular (acneiform) rash. ${ }^{3}$ However, there is a low incidence of some adverse effects, such as paronychia and facial hypertrichosis. ${ }^{4}$ We have treated a patient experiencing a spectrum of erlotinib-induced cutaneous toxicities, but the patient showed a higher tumor response to therapy.

\section{Case report}

In 2013, a 59-year-old non-smoking woman was diagnosed with a stage IV lung adenocarcinoma with brain metastasis. She was treated with radiotherapy for the brain and lung lesions, and then received six cycles of platinum-based chemotherapy. Chest computed tomography (CT) revealed a decrease in the size of the lesions. Unfortunately, 3 months previously, pulmonary metastasis was found via CT (Figure 1A). Because a genetic test showed exon 19 deletion, she started receiving erlotinib $(150 \mathrm{mg})$ daily monotherapy.
Correspondence: Jinming Yu Department of Radiation Oncology, Shandong Cancer Hospital and Institute, Jinan, Shandong Province 250II7, People's Republic of China Tel +8653 I 87984729

Fax +8653187984079

Email sdyujinming@163.com 

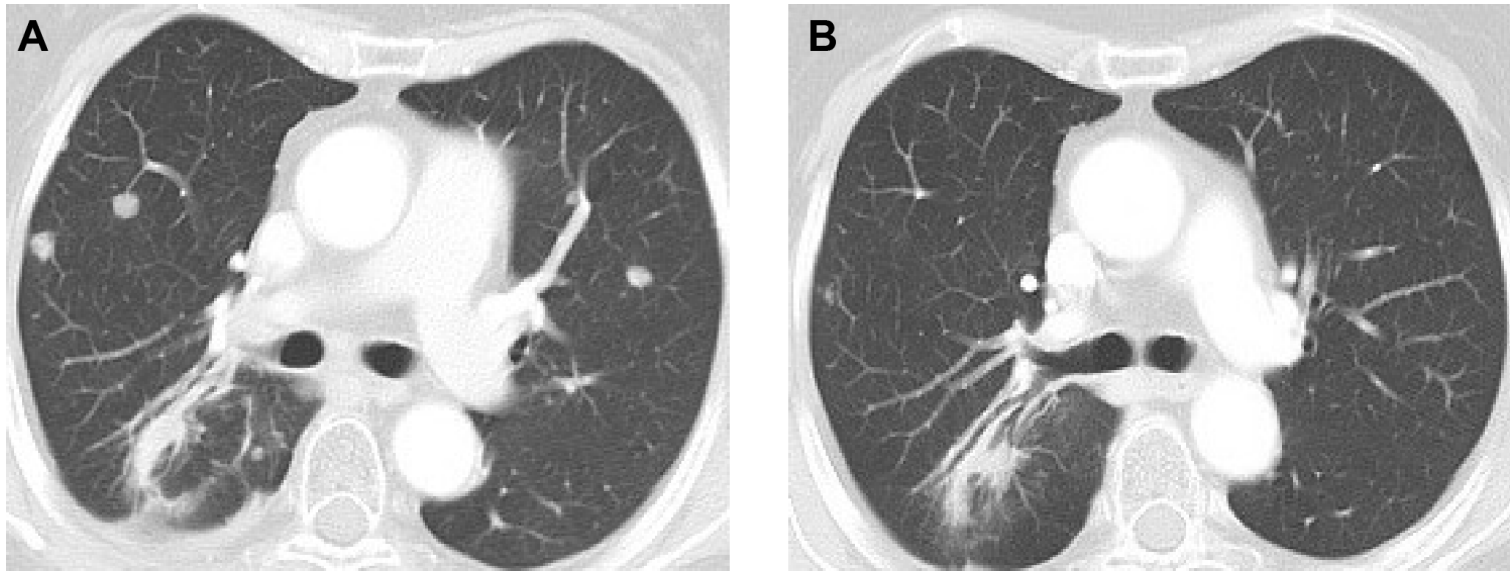

Figure I Chest computed tomographic scan before treatment (A) and after 3 months' treatment (B).

Within 3 weeks of initiating therapy, she experienced cutaneous rashes on her face and upper trunk, cutaneous xerosis and pruritus, mild diarrhea, and mucositis, which were adequately controlled with topical therapy. However, after 2 months of treatment, she developed paronychia of the right middle finger and left ring finger (Figure 2A), accompanied by onychorrhexis. Papulopustular rashes subsequently appeared on the patient's right side of the neck and right leg (Figure 2B), her eyebrows gradually became thicker and more rigid
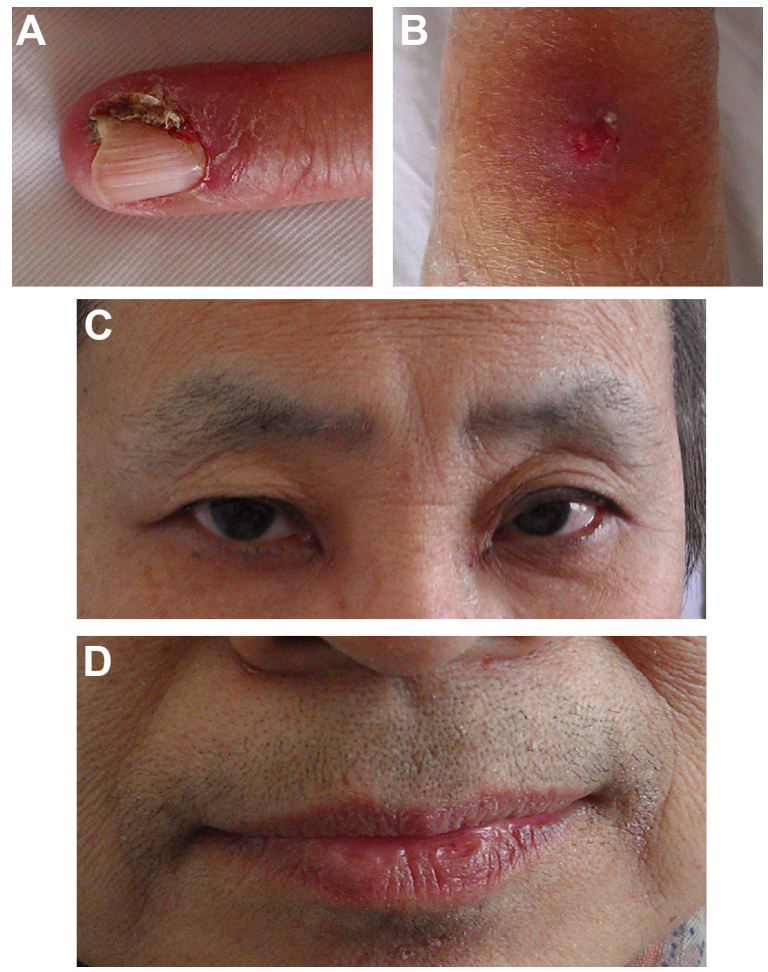

Figure 2 Paronychia in left ring finger (A), papulopustular rash in right leg (B), the thicker eyebrows (C), the darker beard (D) were found, respectively.
(Figure 2C), and she developed the darker beard (Figure 2D). No changes were observed in other parts of the body. During the whole treatment, she did not receive, use, or consume any other medication, and her family history was unremarkable. Laboratory tests did not reveal abnormalities in the levels of her sex hormone. Interestingly, her restaging CT scan showed that the pulmonary metastatic lesions showed almost complete response to the treatment (Figure 1B), and her primary lung tumors and brain metastases showed stable disease.

\section{Discussion}

EGFR is mainly expressed by basal keratinocytes and the outer root sheath cells. ${ }^{4}$ EGFR functions like a switch that is turned on and off in hair follicles at the beginning and end of the anagen phase of the hair cycle. EGFR stimulates proliferation, regeneration, and development in normal cells at the level of the skin, hair follicles, lacrimal gland, and ocular surface epithelia. ${ }^{5}$ Moreover, EGFR is overexpressed or dysregulated in various types of solid tumors, including NSCLC. ${ }^{6}$ This can lead to increased or uncontrolled proliferation, decreased apoptosis, enhanced tumor cell motility, and angiogenesis. Inhibition of EGFR signaling in epidermal and follicular keratinocytes results in decreased cell proliferation, differentiation, and attachment. ${ }^{5}$

Although the underlying mechanism of cutaneous toxicities remains elusive, EGFR inhibitors, such as erlotinib, gefitinib, cetuximab, and panitumumab, are related to cutaneous toxicity. ${ }^{7}$ The selective HER2 inhibitor trastuzumab induces tufted hair folliculitis in some cases. ${ }^{8}$ Cutaneous reactions can be a positive predictor of tumor response, which is believed to indicate receptor saturation and blockade. ${ }^{9}$ This may be one of the mechanisms of erlotinib-induced cutaneous toxicity. Our patient developed a spectrum of cutaneous 
toxicities, including papulopustular rash, mucositis, pruritus, xerosis, paronychia, and facial hirsutism, which is defined as PRIDE syndrome. ${ }^{10}$ The pathogenesis of these symptoms is largely unknown, but there is evidence that this is directly linked to EGFR inhibition. ${ }^{9}$

Because the prognosis of advanced NSCLC is poor and the cost of erlotinib is high, it is imperative that molecular or clinical markers are identified to stratify potential responders. Currently, clinicians mainly evaluate the efficiency of erlotinib treatment using EGFR mutation analysis. Several factors, such as female sex, never smokers, adenocarcinomas (particularly bronchoalveolar adenocarcinoma), and Asian origin, are established as positive predictive markers for clinical benefit. ${ }^{11}$ For Caucasian patients, never-smoking status and male sex predicted a prolonged survival. ${ }^{12}$ However, sometimes it is not possible to obtain the tumor genotypes owing to tumor characteristics or technical problems. Therefore, there is a need to indirectly speculate tumor response through clinicopathological characteristics.

Several studies have reported a link between the antitumor efficacy of EGFR inhibitors and cutaneous adverse effects. The earliest literature suggests that the rash can be used as a marker of EGFR inhibitor treatment efficiency. ${ }^{13}$ Recently, a meta-analysis showed that patients with skin rash have a longer overall survival than patients without skin rash. ${ }^{11}$ There are also data suggesting that skin changes may be a surrogate marker for the response of a tumor to EGFR inhibition, and that skin rash is a prognostic factor of patients with NSCLC. Some researchers have reported that patients with erlotinib-induced trichomegaly had a good tumor response, suggesting that it could be used as an effective tool for predicting drug efficacy. ${ }^{14}$ Recently, it was suggested that cutaneous leukocytoclastic vasculitis may be due to erlotinib and bevacizumab and could be used as a marker of anti-tumor efficacy of EGFR inhibitors. ${ }^{15}$

Currently erlotinib-induced cutaneous toxicities that have been reported as clinical markers are mostly single adverse events. This is the first case report about the relationship between multiple cutaneous adverse effects and EGFR inhibitor efficacy. A study showed that patients experiencing multiple cutaneous toxicities had better therapeutic outcome compared to those experiencing single cutaneous adverse events. ${ }^{16}$ The variability in skin rash susceptibility was associated with erlotinib plasma concentrations, which predicted a good response to erlotinib. ${ }^{17}$ When our patient developed the spectrum of cutaneous reactions, the CT scan showed marked disappearance of the lung metastases. We speculate that multiple cutaneous toxicities indicate a good tumor response. Thus, multiple cutaneous toxicities may be a clinical marker for predicting the response to therapy without knowledge of the EGFR mutation status.

\section{Conclusion}

Overall, the present case suggests that some NSCLC patients who develop a spectrum of cutaneous toxicities might have a good tumor response using erlotinib monotherapy. Our findings provide a method for clinicians to predict erlotinib efficacy in NSCLC therapy without knowledge of the EGFR mutation status. At a time when treatment is increasingly targeting tumor types and patients, exploring the connection between cutaneous toxicities and tumor response may aid in the identification of new clinical markers for treatment efficacy. Currently, for most of the reported studies, the primary outcome parameter was either response to therapy or overall survival. Reporting of toxicities was a secondary aim; thus, data collection has largely been obtained secondarily. ${ }^{18}$ Therefore, there is lack of data in the literature on the frequency of cutaneous toxicities (in general) in non-responders compared to responders to EGFR inhibitor therapy. Future large-scale studies are needed to validate these findings.

\section{Disclosure}

The authors have no conflict of interest to disclose.

\section{References}

1. Lucchini E, Pilotto S, Spada E, Melisi D, Bria E, Tortora G. Targeting the epidermal growth factor receptor in solid tumors: focus on safety. Expert Opin Drug Saf. 2014;13(5):535-549.

2. Gutzmer R, Wollenberg A, Ugurel S, Homey B, Ganser A, Kapp A. Cutaneous side effects of new antitumor drugs: clinical features and management. Dtsch Arztebl Int. 2012;109(8):133-140.

3. Lacouture ME, Anadkat MJ, Bensadoun RJ, et al. Clinical practice guidelines for the prevention and treatment of EGFR inhibitorassociated dermatologic toxicities. Support Care Cancer. 2011;19(8): 1079-1095

4. Robert C, Soria JC, Spatz A, et al. Cutaneous side-effects of kinase inhibitors and blocking antibodies. Lancet Oncol. 2005;6(7): 491-500.

5. Borkar DS, Lacouture ME, Basti S. Spectrum of ocular toxicities from epidermal growth factor receptor inhibitors and their intermediateterm follow-up: a five-year review. Support Care Cancer. 2013;21(4): 1167-1174.

6. Smith J. Erlotinib: small-molecule targeted therapy in the treatment of non-small-cell lung cancer. Clin Ther. 2005;27(10):1513-1534.

7. Heidary N, Naik H, Burgin S. Chemotherapeutic agents and the skin: An update. J Am Acad Dermatol. 2008;58(4):545-570.

8. Rosman IS, Anadkat MJ. Tufted hair folliculitis in a woman treated with trastuzumab. Targ Oncol. 2010;5(4):295-296.

9. Keefe DM, Bateman EH. Tumor control versus adverse events with targeted anticancer therapies. Nat Rev Clin Oncol. 2012;9(2): 98-109.

10. Lacouture ME, Lai SE. The PRIDE (papulopustules and/or paronychia, regulatory abnormalities of hair growth, itching, and dryness due to epidermal growth factor receptor inhibitors) syndrome. Br J Dermatol. 2006;155(4):852-854 
11. Liu HB, Wu Y, Lv TF, et al. Skin rash could predict the response to EGFR tyrosine kinase inhibitor and the prognosis for patients with non-small cell lung cancer: a systematic review and meta-analysis. PLoS One. 2013;8(1):e55128.

12. Faehling M, Eckert R, Kuom S, Kamp T, Stoiber KM, Schumann C. Benefit of erlotinib in patients with non-small-cell lung cancer is related to smoking status, gender, skin rash and radiological response but not to histology and treatment line. Oncology. 2010;78(3-4):249-258.

13. Perez-Soler R. Can rash associated with HER1/EGFR inhibition be used as a marker of treatment outcome? Oncology (Williston Park). 2003; 17(11 Suppl 12):23-28.

14. Carser JE, Summers YJ. Trichomegaly of the eyelashes after treatment with erlotinib in non-small cell lung cancer. J Thorac Oncol. 2006;1(9): 1040-1041.
15. Brandi G, Venturi M, Dika E, Maibach H, Patrizi A, Biasco G. Cutaneous leukocytoclastic vasculitis due to erlotinib: just an adverse event or also a putative marker of drug efficacy. Cutan Ocul Toxicol. 2013; 32(4):336-338.

16. Chanprapaph K, Vachiramon V, Rattanakaemakorn P. Epidermal growth factor receptor inhibitors: a review of cutaneous adverse events and management. Dermatol Res Pract. 2014;2014:734249.

17. Rudin CM, Liu W, Desai A, et al. Pharmacogenomic and pharmacokinetic determinants of erlotinib toxicity. J Clin Oncol. 2008;26(7): 1119-1127.

18. Bentzen SM, Trotti A. Evaluation of early and late toxicities in chemoradiation trials. J Clin Oncol. 2007;25(26):4096-4103.

\section{Publish your work in this journal}

OncoTargets and Therapy is an international, peer-reviewed, open access journal focusing on the pathological basis of all cancers, potential targets for therapy and treatment protocols employed to improve the management of cancer patients. The journal also focuses on the impact of management programs and new therapeutic agents and protocols on

\section{Dovepress}

patient perspectives such as quality of life, adherence and satisfaction. The manuscript management system is completely online and includes a very quick and fair peer-review system, which is all easy to use. Visit http://www.dovepress.com/testimonials.php to read real quotes from published authors. 